\title{
Parkinson's Disease: A Case Report of Motor Symptoms Resolution Following Antibiotic Treatment for Suspected Bacterial Osteomyelitis
}

\author{
Leheste JR ${ }^{1 *}$, Gottlieb SF ${ }^{1}$, Biegel CA$^{1}$, Ramos RL1, Torres $\mathbf{G}^{3}$ and Saggio $\mathbf{G}^{2}$
}

${ }^{1}$ Department of Biomedical Sciences, New York Institute of Technology College of Osteopathic Medicine, Old Westbury, New York, USA ${ }^{2}$ Department of Clinical Specialties, New York Institute of Technology College of Osteopathic Medicine, Old Westbury, New York, USA

${ }^{3}$ Counseling and Psychology Program, Medaille College, Buffalo, New York, USA

\begin{abstract}
Background and case: The vast majority of Parkinson's disease (PD) cases occur sporadically without any obvious etiological schema. That poses major hurdles to the discovery of effective preventative and treatment strategies. Here we are reporting the case of an 86-year-old Caucasian male, with a 21-year history of PD, who reports complete resolution of PD - related motor symptoms after treatment with antibiotics for suspected bacterial osteomyelitis.

Results: After a right leg injury, the patient was prescribed a ten-day course of ciprofloxacin and a four-week course of dicloxacillin for suspected osteomyelitis. After completion of the antibiotics, the patient reported his pervasive motor symptoms as "completely resolved" which to this day have not returned.

Conclusion: PD is a progressive, mostly idiopathic, neurodegenerative disorder with effective but limited treatment options and without compelling preventative strategies. Here we identify the first case indicating the resolution of PD-linked motor symptoms following an unrelated treatment course with antibiotics. This raises interesting questions about the use of antibiotics in PD and the potential of an etiological bacterial connection. The microbiome signature of PD is a current 'hot topic' of investigation and studies need to continue investigating bacteria as a possible causes of PD and antibiotics as effective treatment modalities.
\end{abstract}

Keywords: Antibiotics; Parkinson's disease; Dicloxacillin; Dopamine; Neuroinflammation

\section{Introduction}

An 86-year-old Caucasian male, with a 21-year history of Parkinson's Disease (PD), reports complete resolution of PD-related resting tremors after treatment with antibiotics for suspected bacterial osteomyelitis.

\section{Case Report}

In 1997, a 70-year-old Caucasian male, a retired Physician's Assistant, presented to his neurologist for worsening limp and dragging of his left foot. His gait became worse when he was fatigued. Six months later, he developed a left upper extremity resting tremor that improved with posture and intention and worsened with anxiety. On physical exam, he had 5/5 motor strength in all four extremities with no cogwheeling or paratonia, no pronator drift, $2+$ reflexes, no Babinski, normal proprioception, rapid repetitive finger movement with fingerto-nose movements, movement initiation, posture and steppage gait and $2+$ pedal pulses with no pedal edema. Pertinent positives were decreased vibratory sensation in both feet, resting left upper extremity tremor that dissipates with intention and posture, absence of left arm swing with ambulation and does not turn en bloc. An MRI of the brain and routine lab work revealed no abnormalities. He was diagnosed with early PD and given a trial of Selegiline, which he took for one month and stopped due to minimal symptom improvement.

Over the next two years, the tremor progressed and started to include his left leg in addition to his left arm. He developed a stooped posture and started to notice difficulty with balance and loss of dexterity for fine movements in his left hand. In 2003, he was started on Ropinirole and carbidopa/levodopa. While the dynamics of disease progression and adequate levodopa response did not suggest otherwise, atypical PD cannot be fully ruled out.
In 2009, the patient hit his right leg while using a sledge which resulted in a round dent in his leg (about $3^{\prime \prime} \times 3^{\prime \prime}$ ) with crushed bone. He was prescribed a ten-day course of ciprofloxacin and a four-week course of dicloxacillin for suspected bacterial osteomyelitis. After completion of the antibiotics, the patient reported that his motor symptoms had "completely resolved," with no change in his Parkinson's medications. At the time of writing this manuscript, the patient is now 86 years old and is overall still symptom-free with the occasional exception of a brief resting tremor due to arousal (e.g. excitement, anxiety).

\section{Discussion}

$\mathrm{PD}$ is the most common neurodegenerative motor disorder with the highest crude prevalence across all ages and per 100,000 in Europe (66-1,500) followed by North America (111-329) and South America (31-470) with Asian, African and Arabic countries ranging significantly lower. Worldwide, the incidence proportion lies between 10-18 per 100,000 person-years and depends on factors such as age, ethnicity and gender with males being at higher risk than females (3:2 ratio) [1]. The classical motor symptoms of $\mathrm{PD}$ are caused by degenerating dopamine (DA) producing neurons in the substantia nigra pars compacta ( $\mathrm{SNpc}$ )

*Corresponding author: Dr. Joerg R. Leheste, Department of Biomedical Sciences, New York Institute of Technology College of Osteopathic Medicine, Old Westbury, New York, USA, Tel: +1 516-686-3700; E-mail: jleheste@nyit.edu

Received February 17, 2018; Accepted February 26 2018; Published March 02 2018

Citation: Leheste JR, Gottlieb SF, Biegel CA, Ramos RL, Torres G, et al. (2018) Parkinson's Disease: A Case Report of Motor Symptoms Resolution Following Antibiotic Treatment for Suspected Bacterial Osteomyelitis. J Mol Genet Med 12: 328 doi:10.4172/1747-0862.1000328

Copyright: (c) 2018 Leheste JR, et al. This is an open-access article distributed under the terms of the Creative Commons Attribution License, which permits unrestricted use, distribution, and reproduction in any medium, provided the original author and source are credited 
of the midbrain triggering basal ganglia insufficiency. Clinical motor symptoms are heterogeneous and include bradykinesia, rigidity (mainly cogwheel), postural instability, gait impairment and tremor (mainly resting, pill-rolling). Nonmotor symptoms are frequent and often include neuropsychological deficits early on in the disease process and cognitive impairment later on [2-4]. A definitive diagnosis of PD is typically made after a trial of dopaminergic medications produces clear improvement in symptoms [5].

At present, PD effects about 7.5 million people worldwide over the age of $40[6,7]$. While the pathology is well-defined, general etiology and pathogenesis remain obscure except for a small percentage of established familial causes and toxic incidents. That poses major hurdles to the discovery of effective preventative and treatment strategies. Current treatment is still centered on dopaminergic drugs which are initially effective but progressively lose their effectiveness often prompting microelectrode implantation and deep brain stimulation as a last resort $[8,9]$. Despite decades of work and billions of research dollars, effective preventative strategies are still not at hand.

The accumulation of intracellular Lewy bodies - insoluble aggregations of alpha-synuclein protein - as well as the occurrence of neuroinflammation within the central nervous system (CNS) are most commonly observed with the disease [10]. While potential factors for either condition have not yet been defined, some work is pointing to a beneficial role of antibiotics in the process. While one school of thought is focusing on anti-inflammatory mechanisms of action, the other is set on bacteriostatic/bactericidal effects $[11,12]$.

The recent appreciation of the human microbiome in health and disease has initiated a fresh look at the non-genetic etiology of PD and the potential involvement of microbes. Based on the observation that non-motor manifestations, such as anosmia and constipation, often precede the motor symptoms of $\mathrm{PD}$, one mechanism suggested by Braak's dual-pathway hypothesis suggests a disease origin outside of the CNS, specifically in the nasopharynx and gastrointestinal (GI) nerve plexuses [13]. According to this model, there is a relationship between the intestinal microbiome and the enteric nervous system (ENS) in the pathogenesis of PD. Dysbiosis of the intestinal flora can modulate an immune response that disrupts the integrity of the blood-brain barrier (BBB) at the level of the submucosal and myenteric nerve plexuses [14]. This allows microbes that are both foreign and native to the GI tract to penetrate the nervous system, stimulate an inflammatory response, and induce the folding of alpha-synuclein, which has been found in neurons of the ENS [13].

Recent studies suggest, that neuroinflammation of the brain parenchyma can be mediated by bacteria. Two common routes of entry for bacteria into the CNS from the external environment are the olfactory tract and the vagus nerve. It is postulated that bacteria migrate along peripheral nerves in a retrograde fashion similar to certain molecules and viral examples.

Recent research in animal models is now pointing toward the possibility that the precipitation of soluble alpha synuclein into insoluble Lewy bodies may be triggered by dysregulation of the intestinal microflora followed by Lewy body propagation into the CNS along vagal fibers of the gut-brain axis [14]. Thus, neuroinflammation either caused by disturbances in the GI microbiome or by direct bacterial invasion of midbrain structures may potentially lead to PD, especially when dopaminergic neurons of the SNpc are affected [15]. A computational study based on a large Danish patient cohort revealed that full truncal vagotomy is associated with a lower risk of PD [16] suggesting a role for microbes or their metabolites moving along the vagus nerve. Given the nature of this mechanism of neurodegeneration, therapeutic strategies to target PD may include the future use of antibiotics due to their anti-inflammatory and antibacterial properties. The nasal cavity and the olfactory nerve add another weak spot and point of CNS entry for opportunistic microbes. Work conducted in mice shows that certain Salmonella species, when injected intranasally, intraperitoneally or orally, are subsequently found in the brain parenchyma [17]. The study specifically demonstrates, how intranasally injected bacteria enter the cranium through the cribriform plate of the ethmoid bone - a highly perforated bone containing olfactory nerve fibers. Once there, the bacteria colonize the olfactory nerves and bulbs, and migrate along the olfactory tracts towards other brain areas [17].

Microglia, macrophages of the central nervous system, react to bacterial invasion by polarizing to either the M1 or M2 subtypes. The M1 subtype produces substances that promote inflammation, cell death and damage, while the M2 subtype is anti-inflammatory, promoting cell repair. During most bacterial infections, the M1 subtype is activated first to control the spread of bacteria to other areas. Once the infection is contained, the M2 subtype takes over to repair the damage and control excess inflammation. In some cases, dysregulation of the M1-M2 balance occurs, leading to overexpression of M1 and extensive cell damage as a result [18]. Despite the distinct developmental path of microglia which emerge from the yolk sac and not from the fetal liver as other macrophages, M1 activation is a known feature of the PD brain inflammatory component and interestingly tied into Lewy body formation [19].

Our laboratory is the first to have identified the skin acne-causing bacterium, Propionibacterium acnes (P. acnes), inside neurons of the cadaveric PD midbrain [15]. This small, Gram-positive, anaerobic rod bacterium is characterized as benign but known to unfold its full opportunistic potential when gaining entry to privileged spaces where it can cause debilitating disease [20]. P. acnes is extremely slow-growing, able to enter and colonize cells of epithelial origin (e.g. neurons) and evade lysosomal breakdown inside macrophages [21,22]. How this exactly translates into microglial handling and response is currently under investigation but raises the question if $P$. acnes could be the PD pathological agent Braak and colleagues hypothesized [13].

In this case, the patient was treated with two antibiotics immediately before the resolution of his PD symptoms: Ciprofloxacin and dicloxacillin. Ciprofloxacin is a broad-spectrum fluoroquinolone that covers both Gram-positive and Gram-negative bacteria [23]. Dicloxacillin is a narrow spectrum beta-lactam antibiotic of the penicillin class, which covers gram positive bacteria, mainly Staphylococcus aureus [24-26]. While ciprofloxacin has moderate penetration through an uninflamed $\mathrm{BBB}$, penetration drastically increases with inflammation of the meninges. Dicloxacillin also has the ability to penetrate the $\mathrm{BBB}$, and penetration increases with sustained long-term use [27]. It is interesting to speculate whether the 'perfect storm' of meningeal inflammation combined with continued use of Gram positive targeted antibiotics could have led to the eradication of susceptible bacteria, such as $P$. acnes, from the brain of this PD patient.

\section{Conclusion}

$\mathrm{PD}$ is the most common progressive, neurodegenerative motor disease in the world with the vast majority of cases (>90\%) being idiopathic with unclear etiological and pathogenic roots. Dopamine drugs, which have been the standard of care for many years, lose their effectiveness after a few years leaving patients with little options other 
Citation: Leheste JR, Gottlieb SF, Biegel CA, Ramos RL, Torres G, et al. (2018) Parkinson's Disease: A Case Report of Motor Symptoms Resolution Following Antibiotic Treatment for Suspected Bacterial Osteomyelitis. J Mol Genet Med 12: 328 doi:10.4172/1747-0862.1000328

Page 3 of 3

than microelectrode implantation which comes with a host of risks and complications. While working with human cadaveric PD brains, we have found first evidence of a potential bacterial cause of the disease which fits well with this case of complete resolution of PD symptoms following treatment with antibiotics prescribed for suspected bacterial osteomyelitis. The PD community is desperate for new preventative strategies and a cure. A bacterial cause paired with an early detection mechanism, such as Lewy body accumulation in peripheral gut neurons, would open a new chapter for novel preventative and treatment modalities based on antibiotics. At this point, it is too early to declare a causative relationship between antibiotic treatment and the resolution of PD motor symptoms, but it is an intriguing prospect and we are going to follow up.

\section{Acknowledgements}

This work was supported by NIH Grant 1R15GM117501.

\section{References}

1. Kalia LV, Lang AE (2016) Parkinson disease in 2015: Evolving basic, pathological and clinical concepts in PD. Nat Rev Neurol 12: 65-66.

2. Elgh E, Domelloef M, Linder J, Edstroem M, Stenlund H, et al. (2009) Cognitive function in early Parkinson's disease: A population-based study. Eur $\mathrm{J}$ Neurol 16: $1278-1284$

3. Foltynie T, Brayne CE, Robbins TW, Barker RA (2004) The cognitive ability of an incident cohort of Parkinson's patients in the UK. The camPaIGN study. Brain 127: 550-560.

4. Dirnberger G, Jahanshahi M (2013) Executive dysfunction in Parkinson's disease: A review. J Neuropsychol 7: 193-224.

5. Gelb DJ, Oliver E, Gilman S (1999) Diagnostic criteria for Parkinson disease. Arch Neurol 56: 33

6. Marras C, Tanner CM (2004) Epidemiology of Parkinson's Disease. In: Movement Disorders: Neurologic Principles and Practice (2nd edn), Watts RL, Koller WC (Eds), The McGraw-Hill Companies, New York, USA.

7. Lang AE, Lozano AM (1998) Parkinson's disease. First of two parts. N Engl J Med 339: 1044.

8. Connolly BS, Lang AE (2014) Pharmacological treatment of Parkinson disease: A review. JAMA 311: 1670

9. Constant JP, Fraley GS, Forbes E, Hallas BH, Leheste JR, et al. (2012) Resveratrol protects neurons from cannulae implantation injury: Implications for deep brain stimulation. Neurosci 222: 333-342.

10. Wakabayashi K, Tanji K, Mori F, Takahashi H (2007) The Lewy body in Parkinson's disease: Molecules implicated in the formation and degradation of alpha-synuclein aggregates. Neuropathology 27: 494.
11. Tauber SC, Nau R (2008) Immunomodulatory properties of antibiotics. Curr Mol Pharmacol 1: 68-79.

12. Sadarangani SP, Estes LL, Steckelberg JM (2015) Non-anti-infective effects of antimicrobials and their clinical applications: A review. Mayo Clin Proc 90: 109-127.

13. Choudhry H, Perlmuter L (2017) Non-CNS pathogenic origin of Parkinson's disease. J Neurol 264: 2027-2030.

14. Mulak A, Bonaz B (2015) Brain-gut-microbiota axis in Parkinson's disease. World J Gastroenterol 21: 10609-10620.

15. Leheste JR, Ruvolo KE, Chrostowski JE (2017) P. acnes - Driven Disease Pathology: Current Knowledge and Future Directions. Front Cell Infect Microbiol 7: 81.

16. Svensson E, Horváth-Puhó E, Thomsen RW, Djurhuus JC, Pedersen L, et al. (2015) Vagotomy and subsequent risk of Parkinson's disease. Ann Neurol 78: 522-529.

17. Bollen WS, Gunn BM, Mo H, Lay MK, Curtiss R (2008) Presence of wild-type and attenuated Salmonella enterica strains in brain tissues following inoculation of mice by different routes. Infect Immun 76: 3268-3272.

18. Michels M, Sonai B, Dal-pizzol F (2017) Polarization of microglia and its role in bacterial sepsis. J Neuroimmunol 303: 90-98.

19. Moehle MS, West AB (2015) M1 and M2 immune activation in Parkinson's Disease: Foe and ally? Neurosci 302: 59-73.

20. Perry A, Lambert P (2011) Propionibacterium acnes: Infection beyond the skin Expert Rev Anti Infect Ther 9: 1149-1156.

21. Mak TN, Fischer N, Laube B (2012) Propionibacterium acnes host cell tropism contributes to vimentin-mediated invasion and induction of inflammation. Cell Microbiol 14: 1720-1733.

22. Fischer N, Mak TN, Shinohara DB, Sfanos KS, Meyer TF, et al. (2013) Deciphering the intracellular fate of Propionibacterium acnes in macrophages. Biomed Res Int 2013: 603046.

23. Drlica K, Zhao X (1997) DNA gyrase, topoisomerase IV, and the 4-quinolones Microbiol Mol Biol Rev 61: 377-392.

24. Spratt BG, Cromie KD (1998) Penicillin-binding proteins of gram-negative bacteria. Rev Infect Dis 10: 699-711.

25. Novak R, Charpentier E, Braun JS, Tuomanen E (2000) Signal transduction by a death signal peptide: Uncovering the mechanism of bacterial killing by penicillin. Mol Cell 5: 49-57.

26. Mulligan ME, Murray-leisure KA, Ribner BS (1993) Methicillin-resistant Staphylococcus aureus: A consensus review of the microbiology, pathogenesis, and epidemiology with implications for prevention and management. Am J Med 94: 313-328.

27. Nau R, Sörgel F, Eiffert H (2010) Penetration of drugs through the bloodcerebrospinal fluid/blood-brain barrier for treatment of central nervous system infections. Clin Microbiol Rev 23: 858-883. 